\title{
Siedlungs- und Industrieentwicklung im Birrfeld
}

Peter Abt und Hans Elsasser

\section{Einleitung}

Während der 107. Jahresversammlung des Vereins Schweizerischer Gymnasiallehrer (13./14. November in Baden) führten die Geographen eine Exkursion ins Birrfeld durch. Diese war als Fortsetzung der vor zwei Jahren veranstalteten Badner Planungsexkursion gedacht. Wiederum zeigten zahlreiche Teilnehmer reges Interesse an den aktuellen Problemen der Siedlungs- und Industrieentwicklung in der Region Baden-Brugg. Dank dem freundlichen Entgegenkommen der AG Brown, Boveri \& Cie. (BBC) und der Gemeindebehörde Birr durften wir anhand dreier Referate und einer anschließenden Werkbesichtigung einen vorzüglichen Einblick in die Entwicklung der Werkanlagen Birrfeld nehmen. Im folgenden sollen die wesentlichsten Punkte kurz zusammengefaßt werden.

Die jüngste Geschichte des Birrfeldes ist eng verknüpft mit der Entwicklung der BBC. Drei hauptsächliche Faktoren führten zu einer bedeutenden Entfaltung des Konzerns. Erstens nimmt der Energieverbrauch weltweit zu. Zweitens macht die Elektrotechnik bedeutende Fortschritte. Drittens ist die Entwicklung in der Fabrikationstechnik festzuhalten. Durch die Automation und die zunehmende Größe der Werkstücke steigen benötigte Fläche und Energieausstattung je Mann in der Werkstatt an. Die Platzknappheit in Baden veranlaßte die BBC, sich nach neuen Industriestandorten umzusehen. Von den Varianten Birr, Turgi und Würenlingen sind die beiden erstgenannten realisiert worden (Elektronik in Turgi). Für das Birrfeld begann damit ein tiefgreifender Landschafts- und Strukturwandel.

\section{Die Industriezone Birr}

Das Birrfeld galt seit jeher als Kornkammer des Kantons Aargau. Die Birrfeldgemeinden waren ursprünglich reine Bauerngemeinden. Der Anteil des offenen Ackerlandes beträgt zum Beispiel in der Gemeinde Birr über 50 Prozent der landwirtschaftlichen Nutzfläche (1965). Nach dem Zweiten Weltkrieg beschlossen die Gemeinden des Birrfeldes eine Integralmelioration. Große Flurblöcke anstelle der einstigen verzettelten Kleinparzellen sowie ein vollständig neu angelegtes Netz von Wegen und Güterstraßen schufen die Voraussetzungen für eine mo- derne Landwirtschaft. Doch diese Neugruppierung leitete eine Entwicklung ein, an die man zunächst nicht gedacht hatte: die Festsetzung von Industrieunternehmen. Mitte der fünfziger Jahre, also gerade zu der Zeit, da die Güterzusammenlegung beendet war, begann sich die BBC für potentielles Industrieland $\mathrm{zu}$ interessieren. Úber eine mehrjährige Zeitspanne hinweg tätigte diese Firma Landkäufe im Gemeindegebiet von Birr. 1955 mußte für einen Quadratmeter Boden ein Preis von rund 4 Franken bezahlt werden. Heute werden Quadratmeterpreise von über 60 Franken notiert. Damals verfügte Birr noch über keine Bauordnung. Erst im Jahre 1957 wurde eine solche erlassen. Dabei wurde eine Industriezone von rund 64 ha Fläche ausgeschieden. Davon befinden sich heute nicht weniger als 52 ha im Besitz der BBC, währenddem die übrigen 12 ha den Kabelwerken Brugg gehören.

$\mathrm{Da} ß$ sich die $\mathrm{BBC}$ gerade im Birrfeld umfangreiche Landreserven gesichert hat, ist auf das Zusammentreffen einer ganzen Reihe von sehr guten Standortvoraussetzungen in diesem Gebiet zurückzuführen: Beim Birrfeld handelt es sich um ein in topographischer Hinsicht für industrielle Nutzung vorzüglich geeignetes Gelände. Das gesamte Gebiet weist praktisch keinerlei Neigung auf. Auch die Baugrundverhältnisse dürfen als sehr gut beurteilt werden. Ferner enthält die große Schotterfläche in verschiedenen Stockwerken erhebliche Grundwasservorkommen. Bereits zu Beginn der Industriezonenerschließung erstellte die BBC ein betriebseigenes Pumpwerk. Dessen Kapazität wurde so groß dimensioniert, daß die Firma über die eigene Bedarfsdeckung hinaus noch in der Lage ist, Drittinteressierten Wasser abzugeben. Daneben besteht auch noch ein gemeindeeigenes Pumpwerk. Gegenwärtig werden weitere Versuchsbohrungen durchgeführt, damit der Platz eines künftigen Wasserwerkes mit einer möglichen Grundwasseranreicherungsanlage heute schon ermittelt und gesichert werden kann. Eine Grundvoraussetzung für die industrielle Úberbauung des Birrfeldes war die Lösung des Abwasserproblems. Da das Birrfeld von keinem Fließwasser durchlaufen wird, welches als Vorfluter dienen könnte, bot die Entsorgung die größten Probleme und verursachte die größten Aufwendungen bei der Erschließung. Im Rahmen eines Zweckverbandes wurde ein Sammelkanal vom Birrfeld bis zur neu errichteten Kläranlage im Win- 
discher Schachen erstellt. Dieser mußte in seinen Dimensionen auf den Endausbau des heute eingezonten Baugebietes ausgerichtet sein. Von maßgebender betriebswirtschaftlicher Bedeutung ist aber auch die außerordentlich günstige verkehrsmäßige Lage des Birrfeldes. Zunächst ist auf die beiden Autobahnanschlüsse hinzuweisen, welche in geringer Distanz vom nördlichen beziehungsweise südlichen Rand des Industriegebietes liegen oder projektiert sind. Die Industriezone Birr ist somit direkt an die Nationalstraßen Bern-Zürich und BaselZürich angeschlossen. In Nord-Süd-Richtung ist eine Erschließungsstraße projektiert, einerseits als Trennung der Industriezone von der Wohnzone und anderseits als wichtiger Verkehrsträger zwischen dem nördlichen und südlichen Autobahnanschluß. Neben der vorzüglichen straßenorientierten Erschließung verfügt dieses Gebiet aber auch über eine ebensogute bahnmäßige. Durch den Bau des Werkes Birr mit seinen rund 2000 neuen Arbeitsplätzen entstand ein erheblicher Bedarf an Wohnraum. Diesem stand in der näheren Umgebung kein genügendes Angebot gegenüber. $\mathrm{BBC}$ mußte sich deshalb bei der Projektierung der Industrieanlagen gleichzeitig auch mit der Siedlungsplanung befassen. Die Firma erwarb rund 22 ha Wohngebiete in der Region des Birrfeldes. 1959 wurde mit der Projektierung für eine Mehrfamilienhausüberbauung, der Uberbauung "Wyden", auf einem in unmittelbarer Nähe des Werkes gelegenen Grundstück von 6,7 ha Fläche begonnen. Die Lage des Baugeländes bringt es mit sich, daß die Siedlung vorerst ein eigentliches, vom bestehenden Dorf Birr losgelöstes neues Zentrum darstellt, welches sich später in die «neue Stadt Birrfeld» einfügen soll. Die Úberbauung umfaßt zwei leicht geknickte $150 \mathrm{~m}$ lange Wohnhaustrakte auf den beiden Längsseiten und wird durch je drei würfelförmige Wohnbauten sowie durch ein Einkaufszentrum mit Restaurant auf den Schmalseiten locker abgeschlossen. Innerhalb der Uberbauung liegt ferner ein Kindergarten. In der Siedlung mit ihren 530 Wohnungen $(20 \%$ Kleinwohnungen, $50 \% \quad 3 \frac{1}{2}$-Zimmer-Wohnungen und $30 \%$ Wohnungen mit $4 \frac{1}{2}$ und $5 \frac{1}{2}$ Zimmern) leben rund 2000 Personen.

Mit Ausnahme einiger weniger höherer Angestellten wohnen vorläufig (noch) keine leitende Betriebsanghörige in Birr. Dafür sind verschiedene Gründe maßgebend: Zunächst verfügt die $\mathrm{Ge}$ meinde nur über eine sehr rudimentäre sozio-kulturelle Infrastruktur; eine Bezirksschule ist nicht mehr vorhanden. Außerdem fehlt in Birr eine topographisch günstige Wohnlage, im besonderen kann kein Südhang angeboten werden. Im Zonenplan finden wir deshalb auch keine Landhaus-, sondern lediglich eine Einfamilienhauszone. Endlich muß noch auf einen für die Industrie sehr bedeutsamen Standortfaktor hingewiesen werden: die außeror- dentlich beweglichen Vorschriften für die Industriezone der Einwohnergemeinde Birr. Dabei steht der folgende Passus im Mittelpunkt: «Die zulässige Höhe der Bauten sowie die Grenz- und Gebäudeabstände und weitere Bauvorschriften werden vom Gemeinderat in Abwägung der öffentlichen und privaten Interessen von Fall zu Fall festgelegt ... » Die Möglichkeit einer derart flexiblen Ausgestaltung der Zonenvorschriften fußt auf dem Baugesetz des Kantons Aargau, welches noch aus dem vorigen Jahrhundert stammt. In anderen Kantonen haben sich die Gemeinden teilweise an einschränkendere kantonale Bauvorschriften zu halten. Das Baugesetz des Kantons Zürich (Baugesetz für Ortschaften mit städtischen Verhältnissen) beispielsweise statuiert eine maximal zulässige Bauhöhe von $20 \mathrm{~m}$, welche nur mit spezieller Bewilligung des Regierungsrates überschritten werden darf.

Abschließend soll noch ein Blick auf die Ertragsseite des Kommunalhaushaltes geworfen werden. Zunächst fällt die gewaltige Bevölkerungszunahme der Gemeinde Birr seit 1960 auf (vgl. Tabelle Bevölkerungsentwicklung). Trotzdem ist der Steuerertrag der natürlichen Personen - im Verhältnis zu den entsprechenden Aufwendungen - nicht sehr bedeutend, weil sich keine Steuerpflichtigen mit hohen Einkommen darunter befinden. Das Hauptaugenmerk muß sich wohl auf den Steuerertrag der juristischen Personen richten. BBC hat ihren Betrieb im Birrfeld 1961 aufgenommen. Von den 52 ha, dem gesamten Areal dieser Firma, werden heute lediglich 10 bis 15 ha direkt industriell genutzt. Das übrige Gelände dient der Firma als Landreserve auf lange Sicht. Das BBC-Steuerdomizil befindet sich nach wie vor in Baden. Eine Sitzverlegung oder eine Neubegründung desselben für den Betrieb Birrfeld konnte die Gemeinde mangels aktiver Bodenpolitik nicht erreichen. Der BBCFiskalertrag der Gemeinde Birr richtet sich deshalb nach der vom Kanton vorgenommenen Steuerausscheidung und beläuft sich auf rund 350000 Franken, verglichen mit einer Steuerbelastung der Firma in Baden von ungefähr 3,8 Millionen Franken. Ne-

\section{Bevölkerungsentwicklung der Gemeinde Birr}
1850
498 Einwohner
1900448 Einwohner
1920488 Einwohner
1950562 Einwohner
1960728 Einwohner
19652250 Einwohner
19682646 Einwohner

(davon 1356 oder $51 \%$ Ausländer) 
benbei sei noch erwähnt, daß das BBC-Areal im Birrfeld kaum weniger Hektaren umfaßt, als jenes am Stammsitz.

\section{Schlußbemerkung}

$\mathrm{BBC}$ beschäftigt insgesamt Angehörige von $56 \mathrm{Na}$ tionen. So erstaunt es nicht, daß in Birr über $50 \%$ der Einwohner Ausländer sind. Ein Drittel der Bevölkerung ist fremdsprachig, und der Anteil der Schulkinder beträgt 17\% (Mittel Aargau 10-11\%). Dieser einzigartigen Situation steht ein großer Teil der Bevölkerung positiv gegenüber. Natürlich gibt es auch Ausnahmen, besonders ältere Leute vermögen den Umbruch psychisch nicht immer zu verarbeiten. Unter den Neuzuzügern können zwei Gruppen unterschieden werden: einerseits jene, welche nur kurzfristig in Birr bleiben, etwa junge Angestellte in der Ausbildung oder solche, die BBC als Sprungbrett für einen andern Arbeitsplatz benützen: sie alle haben keine Zeit oder kein Interesse, sich aktiv am am Gemeindeleben zu beteiligen. Anderseits jene, die sich zu einem längeren Aufenthalt ent- schlossen haben, z. B. Besitzer von Einfamilienhäusern, aber auch zahlreiche Bewohner der Siedlung "In den Wyden». Bereits finden wir einige von ihnen im Gemeinderat, in der Schul- oder Kirchenpflege, in der Kulturkommission. Abgesehen von der Überarbeitung der Ortsplanung Birr und der Realisierung dringender Bauvorhaben, sind es vor allem diese Probleme der zwischenmenschlichen Beziehungen, die es in Zukunft in Birr zu lösen gilt.

\section{Literatur}

Bauordnung der Gemeinde Birr (mit Zonenplan) vom 18. Januar 1957.

Einwohnergemeinde Birr (Herausg.): Birr, Portrait einer aargauischen Gemeinde 1969.

Brown, Boveri (Herausg.): Wohnbauten «In den Wyden», Birr.

Gutersohn H.: Geographie der Schweiz, Band III Mittelland, 2. Teil, Bern 1969.

Mock H. R., Nydegger H.: Die Industriezone Birrfeld (Aargau). ORL-IG Notiz Nr. 26 (unveröffentlicht) ORL-Institut ETHZ. Zürich 1970. 